\title{
Vascular Endothelial Growth Factor Signaling in Fibrosis: An Ophthalmic Perspective
}

\author{
Matthew McDonald* \\ Faculty of Medical and Health Sciences, University of Auckland, New Zealand
}

*Corresponding author: Matthew McDonald, University of Auckland, 85 Park Road, Grafton, Auckland, 1023, New Zealand

To Cite This Article: Matthew McDonald. Vascular Endothelial Growth Factor Signaling in Fibrosis: An Ophthalmic Perspective. 2020 - 8(3). AJBSR.MS.ID.001275. DOI: 10.34297/AJBSR.2020.08.001275.

Received: 㠿 March 27, 2020; Published: 制 April 07, 2020

\begin{abstract}
Fibrosis is a common condition, transcending all areas of medicine. Its pathophysiology has eluded researchers for decades with no universal cure. The cellular processes which underpin these conditions begin with cellular proliferation, followed by migration, epithelial to mesenchymal transformation, and extracellular matrix contraction. This short review highlights the complexities in the signaling cascade of the key event in fibrosis: epithelial to mesenchymal transition with particular emphasis on the role of vascular endothelial growth factor (VEGF). Other growth factors, mitogens, and markers of fibrosis included in this review are transforming growth factor $\beta$ (beta), platelet-derived growth factor, cadherins, Smads, Slug, Snail, $\alpha$-SMA, and the Rho-kinase group, among others. The ocular lens capsule serves as an ideal model to study this pathophysiology due to its isolation from other organs, avascular environment, and experimental models available. Anti-VEGF treatments are well established for neovascular conditions such as age-related macular degeneration and may therefore form a component of anti-fibrotic treatment in the future. Evidence for reduction of fibrotic markers following VEGF inhibition is revealed last.
\end{abstract}

Keywords: VEGF, Vascular endothelial growth factor, Fibrosis, Lens capsule, Posterior capsule Opacification

\section{Fibrosis}

Fibrosis is a pathological process in which a tissue's architecture is disturbed by excessive fibro connective tissue, typically in response to a reparative or reactive response to a cellular insult $[1,2]$. From metabolic malfunction, ischemia, degeneration, or autoimmune inflammatory processes, fibrosis results from proliferation and Trans differentiation of a cellular phenotype (e.g. epithelial cells) to activated fibroblasts known as my fibroblasts. These cells contribute to 'excessive' production of extra-cellular matrix (ECM) components, resulting in contraction and disruption of tissue architecture [1]. In an effort to resolve the damaged tissue, this complex proliferative process often wreaks havoc, leading to worse survival rates than cancer in the most severe cases (i.e. idiopathic pulmonary fibrosis) [3]. In ophthalmology, this results in loss of vision.

\section{Vascular Endothelial Growth Factor}

Vascular Endothelial Growth Factor (VEGF), also known as Vascular Permeability Factor (VPF), is a family of potent mitogens originally thought to solely stimulate endothelial cells in angiogenesis. Multiple genes translate the five known VEGF subtypes (VEGF-A, VEGF-B, VEGF-C, VEGF-D, and placenta growth factor), leading to differences in molecular structure, biological properties, and regulation [4,5]. Appreciating the gross variability in both the VEGF ligand and its receptor, it is the subtleties in this interaction leading to its differential effects. Its functions range from normal physiological regulation of wound healing, angiogenesis (capillary tube formation of endothelial cells), bone formation, hematopoiesis, to embryological development and morphogenesis. In the eye, VEGF is required for visual function through playing a survival role on Muller cells and photoreceptors, indicating an important role in the maintenance and functioning of the adult retin [6]. VEGF will also drive proliferation, migration, cell survival, and even epithelial to mesenchymal transition $[7,8]$.

\section{Epithelial to Mesenchymal Transition}

In epithelial to mesenchymal transition (EMT), which all fibrotic processes share, epithelial cells experience a multitude of biochemical and morphological changes, disengaging from their 
basal surface and basement membrane to migrate and produce extracellular matrix (ECM) components. The resulting mesenchymal cell leaves the epithelial layer from which it originated and turns myofibroblastic [9].

Recent studies have started to cast the net further in order to identify more compounds involved in fibrosis [10-13]. Plateletderived growth factor (PDGF), epidermal growth factor (EGF), transforming growth factor- $\boldsymbol{\beta}$ (TGF- $\boldsymbol{\beta}$ ), tumor necrosis factor- $\alpha$ (TNF- $\boldsymbol{\alpha}$ ) and fibroblast growth factor (FGF) are potent mitogens in this response, closely connected with VEG [14,15]. These factors increase collagen synthesis, a late marker of cellular matrix deposition, while TGF- $\boldsymbol{\beta}$ induces trans differentiation to myofibroblast phenotype. Metalloproteinase inhibitor 1, fibronectin, and connective tissue growth factor (CTGF) are also induced by VEGF which contribute to myofibroblast differentiation $[16,17]$.

Posterior capsule opacification (PCO) is a common fibrotic condition which occurs after cataract surgery. Lens epithelial cells on the anterior lens capsule remain despite enduring the rigors of surgical trauma after an intraocular lens is inserted. This resilient group of cells then colonizes the previously cell-free posterior capsule to proliferate and undergo fibrotic change (epithelial to mesenchymal transition) which scatters light across the visual axis. In PCO, there is loss of E-cadherin from epithelial cells, a key event in EMT, causing a transformative change to a mesenchymal phenotype, leading to migration of these cells across the visual axis. This occurs with an upregulation of $\mathrm{N}$-cadherin, Vimentin, and $\alpha$-SMA, an important marker for fibroblast-myofibroblast conversion and ECM contraction [18-20]. Factors that bind to the E-cadherin promoter to inhibit its transcriptioninclude the zinc finger proteins Slug and Snail signaling pathway (SNAIL1 and SNAIL2), which induces EMT with zinc-finger E-box-binding (ZEB1/2) and Smads to promote Wntsignaling and subsequent $\alpha$-SMA expression [9]. These early points in EMT destabilize communication between epithelial cells as gap junctions are compromised and desmosomes interrupted. Breast cancer models have shown VEGF to induce Snail expression by inhibiting GSK3 $\beta$ (glycogen synthase kinase-3), which normally cooperates with Akt (protein kinase B) to stabilize Snail activity [21]. SNAIL1, in particular, has also shown to increase VEGF expression as well, implying a mutually dependent relationship in augmenting EMT [8].

TGF- $\beta$, a superfamily of $\boldsymbol{\beta}_{1}, \boldsymbol{\beta}_{2}$, and $\boldsymbol{\beta}_{3}$, known as "master regulators" of fibrogenic response and angiogenesis, generally suppress cell proliferation, induce apoptosis, promote EMT, and cell motility. These isoforms also regulate VEGF, and VEGF has even been reported to induce TGF- $\boldsymbol{\beta}_{1}$ depending on a direct interaction between its signaling proteins, Smads, and members of the Wnt/ B-catenin signaling group [22,23]. T cell factor (TCF) provides two binding sites on the VEGF promoter region to which TGF- $\beta$ binds for transcription, illustrated through absent levels of (TGF$\beta$-induced) VEGF promoter activity after blocking these binding sites. Smad4, a common isoform in TGF- $\beta /$ Smad signaling, will trans locate to the nucleus of lens epithelium as soon as $2-12 \mathrm{hrs}$ post-surgical trauma. $\alpha$-SMA is subsequently produced during EMT response. Rho-kinase, PI3-kinase, and Src-family kinases (SFK) also regulate EMT [24-26]. VEGFR-2(KDR/Flk-1) has proven to recruit Src homology 2 domain-containing molecules that lead to activation of MAP kinase signaling. This is significant because p85, a regulatory subunit of PI3-kinase, is strongly associated with Flk-1/KDR; treatment of human umbilical vascular endothelial cells (HUVECs) with VEGF caused tyrosine auto phosphorylation of VEGFR2 which, in turn, caused phosphorylation of p85, showing an increase in PI 3-kinase activity and implicating a larger role in EMT regulation [27]. It is when the PI-3 kinase/Akt pathway is downregulated that bone morphogenetic protein 2 (BMP2), a member of the TGF- $\beta$ super-family, induces EMT and migration through loss of E-cadherin [28]. This hypothesis is consistent with reduced $\alpha$-SMA in sub conjunctival layers of the eye post-anti-VEGF treatment after trabeculectomy (a form of glaucoma surgery) compared to control [22].

\section{The Lens Capsule as a Model for VEGF's Role in Fibrosis}

Further studies investigated the role of VEGF in the lens, an avascular environment, after an active VEGF-A signaling system was revealed [29]. Assessment of medium from human lens capsular bag cultures demonstrated high levels of VEGF relative to other growth factors. In fact, levels were $>10$ fold more than detected for FGF [30]. It has also been proven that treatment of cells with TGF- $\beta$ , EGF, PDGF, and IL-6 induce VEGF mRNA production, suggesting that paracrine or autocrine release of these compounds works with hypoxic conditions in regulating VEGF. Inflammatory cytokines such as IL- 1 and IL- 6 can even induce VEGF in synovial fibroblasts, hinting at an enormous array of compounds mediating its effects, particularly in inflammatory conditions [31,32]. This may therefore serve as a novel therapeutic target.

VEGF inhibition has been shown to reduce key markers of fibrosis and EMT ( $\alpha$-SMA, FN1, Collagen 1, SNAIL1/2, and MMP2) [11]. Using cell-based contraction assays, VEGF inhibition prevents TGF- $\beta 2$ mediated contraction. Immunocyto chemical analysis shows that anti-VEGF therapy works independent of Smad signaling, which suggests contraction is not entirely due to trans differentiation, but perhaps through interaction of actin with myosin in intracellular stress fiber formation. TGF- $\beta 2$ has proven to induce the Rho/Rho kinase pathway, which also relies on myosin light chain phosphatase (MLCPPase) and myosin light chain kinase (MLCK) in regulating contraction. Likewise, myosin activity is a key regulator for TGF- $\beta$ induced contraction in lens cells [33]. In addition to Rho kinase, ERK signaling (regulated by VEGF) can also 
induce matrix contraction via MLCK [34], JNK, and p38 pathways, all of which are induced by TGF- $\beta 2$ as well [35].

Conversely, TGF- $\beta_{2}$ induced collagen I transcription has been shown to decrease with VEGFR inhibition [11]. There is evidence that Smad3 is key for collagen I synthesis, but also the ras/MEK/ ERK MAP kinase cascade [18]. The anti-fibrotic effects of VEGFR inhibition is likely independent of Smad signaling and instead through inhibition of ras/MEK/ERK MAP kinase.

Evidence suggests that VEGF induces SNAIL expression by inhibiting GSK3 $\beta$ (glycogen synthase kinase-3), which normally cooperates with AKT to stabilize SNAIL activity [36]. Blocking this pathway via VEGF's receptor tyrosine kinases clearly exerts downstream effects on these compounds and prevents regulation of EMT.

Given VEGF's role in an array of signaling pathways and physiology, there is clear evidence of an active biochemical pathway in fibrosis, even in an avascular environment such as the human lens. Anti-VEGF treatments are well established for neovascular ocular diseases (e.g. neovascular age-related macular degeneration) so may serve as a readily available treatment for fibrotic conditions. Future directions in fibrosis research, both in ocular and extraocular environments, should focus on targeted therapy on more than one signaling pathway due to the interdependencies illustrated above.

\section{Acknowledgements}

Professor I.M. Wormstone's mentorship early in my career was invaluable in pursuing further research and clinical training.

\section{Conflict of Interest}

None to declare

\section{References}

1. Eldred JA, LJ Dawes, IM Wormstone (2011) The lens as a model for fibrotic disease. Philos Trans R Soc Lond B Biol Sci 366(1568): 13011319.

2. Friedlander M (2007) Fibrosis and diseases of the eye. J Clin Invest 117(3): 576-586.

3. Laurent GJ, McAnulty RJ, Hill M, Chambers R (2008) Escape from the matrix: multiple mechanisms for fibroblast activation in pulmonary fibrosis. Proc Am Thorac Soc. 5(3): 311-315.

4. Kowanetz M, N Ferrara (2006) Vascular endothelial growth factor signaling pathways: therapeutic perspective. Clin Cancer Res 12(17): 5018-5022.

5. Tischer E, Mitchell R, Hartman T, Silva M, Gospodarowicz D, et al. (1991) The human gene for vascular endothelial growth factor. Multiple protein forms are encoded through alternative exon splicing. Journal of Biological Chemistry 266(18): 11947-11954.

6. Magali Saint-Geniez, Arindel SR Maharaj, Tony E Walshe, B udd A Tucker, Eiichi Sekiyama, et al. (2008) Endogenous VEGF is required for visual function: evidence for a survival role on muller cells and photoreceptors. PLoS One 3(11): e3554.
7. Philipp W, L Speicher, C Humpel (2000) Expression of vascular endothelial growth factor and its receptors in inflamed and vascularized human corneas. Invest Ophthalmol Vis Sci 41(9): 2514-2522.

8. Lamouille S, J Xu, R Derynck (2014) Molecular mechanisms of epithelialmesenchymal transition. Nat Rev Mol Cell Biol 15(3): 178-196.

9. Kalluri R, RA Weinberg (2009) The basics of epithelial-mesenchymal transition. J Clin Invest 119(6): 1420-1428.

10. Baah S, Nagel D, Clough R, Rackow AR, Kottman RM (2019) VEGF DownRegulates OGR1 in Pulmonary Fibrosis and Induces Myofibroblast Differentiation. American Journal of Respiratory and Critical Care Medicine 199(A5327).

11. Eldred JA, Matthew McDonald, Helen S Wilkes, David J Spalton, I Michael Wormstone (2016) Growth factor restriction impedes progression of wound healing following cataract surgery: identification of VEGF as a putative therapeutic target. Scientific Reports 6(1): p. 24453.

12. Britta Maurer, Alfiya Distler, Yossra A Suliman, Renate E Gay, Beat A Michel, et al. (2014) Vascular endothelial growth factor aggravates fibrosis and vasculopathy in experimental models of systemic sclerosis. Ann Rheum Dis. 73(10): p. 1880-1887.

13. Yosuke Hirakawa, Tetsuhiro Tanaka, Masaomi Nangaku (2019) Tipping the Balance from Angiogenesis to Fibrosis in Chronic Kidney Disease. In: Fibrosis in Disease, Molecular and Translational Medicine, J Humana Press, USA.

14. Lee KS, Park SJ, Kim SR, Min KH, Lee KY, et al. (2008) Inhibition of VEGF blocks TGF-beta1 production through a PI3K/Akt signalling pathway. Eur Respir J 31(3): 523-531.

15. Hatamochi A, K Mori, H Ueki, (1994) Role of cytokines in controlling connective tissue gene expression. Archives of Dermatological Research 287(1): p. $115-121$.

16. Kuiper Esther J, Van Nieuwenhoven Frans A, de Smet Marc D, van Meurs Jan C, Tanck Michael W et al. (2008) The angio-fibrotic switch of VEGF and CTGF in proliferative diabetic retinopathy. PloS one 3(7): p. e2675-e2675.

17. Kuiper EJ, Hughes JM, Van Geest RJ, Vogels IM, Roel Goldschmeding, et al. (2007) Effect of VEGF-A on Expression of Profibrotic Growth Factor and Extracellular Matrix Genes in the Retina. Investigative Ophthalmology \& Visual Science 48(9): 4267-4276.

18. Leask A, DJ Abraham (2004) TGF-beta signaling and the fibrotic response. FASEB J 18(7): 816-827.

19. Saika S, Yamanaka O, Sumioka T, Miyamoto T, Miyazaki K, et al. (2008) Fibrotic disorders in the eye: targets of gene therapy. Prog Retin Eye Res 27(2): 177-196

20. Terrell A (2013) B1-INTEGRIN MAY REGULATE EGR1 (EARLY GROWTH RESPONSE 1) WITHIN THE LENS. University of Delaware p. 7-11.

21. Wanami LS (2008) Vascular endothelial growth factor-A stimulates Snail expression in breast tumor cells: implications for tumor progression. Exp Cell Res 314(13): p. 2448-2453.

22. Park HY, JH Kim, CK Park (2013) VEGF induces TGF-beta1 expression and myofibroblast transformation after glaucoma surgery. Am J Pathol 182(6): p. 2147-2154.

23. Clifford RL, K Deacon, AJ Knox (2008) Novel regulation of vascular endothelial growth factor-A (VEGF-A) by transforming growth factor (beta)1: requirement for Smads, (beta)-CATENIN, AND GSK3(beta). J Biol Chem 283(51): p. 35337-35353.

24. Patel A, Sabbineni H, Clarke A, Somanath PR (2016) Novel roles of Src in cancer cell epithelial-to-mesenchymal transition, vascular permeability, microinvasion and metastasis. Life sciences 157: 52-61.

25. Knipe RS, AM Tager, JK Liao (2015) The Rho kinases: critical mediators of multiple profibrotic processes and rational targets for new therapies for pulmonary fibrosis. Pharmacological reviews 67(1): p. 103-117. 
26. Mercer PF, Woodcock HV, Eley JD, Platé M, Sulikowski MG, et al. (2016) Exploration of a potent PI3 kinase/mTOR inhibitor as a novel antifibrotic agent in IPF. Thorax 71(8): 701-11.

27. Hans-Peter Gerber, Amy McMurtrey, Joe Kowalski, Minhong Yan, Bruce A Keyt, et al. (1998) Vascular Endothelial Growth Factor Regulates Endothelial Cell Survival through the Phosphatidylinositol 3'-Kinase/ Akt Signal Transduction Pathway: REQUIREMENT FOR Flk-1/KDR ACTIVATION. Journal of Biological Chemistry 273(46): p. 30336-30343.

28. Yoo Y, Kang MH, Kim JS, Oh SC (2007) Invasion in BMP2-induced EMT is mediated through down-regulation of PI-3 Kinase/Akt pathway. Molecular Cancer Therapeutics 13(11).

29. Shui YB, Wang X, Hu JS, Wang SP, Garcia CM (2003) Vascular Endothelial Growth Factor Expression and Signaling in the Lens. Investigative Ophthalmology \& Visual Science. 44(9): 3911-3919.

30. Dawes LJ, G Duncan, IM Wormstone (2013) Age-related differences in signaling efficiency of human lens cells underpin differential wound healing response rates following cataract surgery. Invest Ophthalmol Vis Sci 54(1): 333-342.

31. Ferrara N (2004) Vascular endothelial growth factor: basic science and clinical progress. Endocr Rev 25(4): 581-611.
32. Berndt Enholm, Karri Paavonen, Ari Ristimäki, Vijay Kumar, Yuji Gunji, et al. (1997) Comparison of VEGF, VEGF-B, VEGF-C and Ang-1 mRNA regulation by serum, growth factors, oncoproteins and hypoxia. Oncogene 14: 2475-2483.

33. Dawes LJ, Sleeman M, Anderson I, Reddan J, Wormstone IM, (2009) TGF $\beta /$ Smad4-Dependent and -Independent Regulation of Human Lens Epithelial Cells. IOVS 50(11): 5318-5327.

34. Richard L Klemke, Shuang Cai, Ana L Giannini, Patricia J Gallagher, Primal de Lanerolle, et al. (1997) Regulation of cell motility by mitogenactivated protein kinase. J Cell Biol 137(2): 481-492.

35. Borbiev T, Birukova A, Liu F, Nurmukhambetova S, Gerthoffer WT, et al. (2004) p38 MAP kinase-dependent regulation of endothelial cell permeability. Am J Physiol Lung Cell Mol Physiol 287(5): 911-918.

36. Luke S Wanami, Hsin-Ying Chen, Sandóra Peir, Antonio García de Herreros, Robin E Bachelder (2008) Vascular endothelial growth factor-A stimulates Snail expression in breast tumor cells: implications for tumor progression. Experimental cell research 314(13): 2448-2453. 